\title{
MiR-215-5p is a tumor suppressor in colorectal cancer targeting EGFR ligand epiregulin and its transcriptional inducer HOXB9
}

Petra Vychytilova-Faltejskova 1,2, Jana Merhautova ${ }^{1,3}$, Tana Machackova', Irene Gutierrez-Garcia ${ }^{4}$, José Garcia-Solano ${ }^{5}$, Lenka Radova', Dominika Brchnelova', Katerina Slaba', Marek Svoboda,2, Jana Halamkova², Regina Demlova ${ }^{3}$, Igor Kiss², Rostislav Vyzula², Pablo Conesa-Zamora ${ }^{4}$ and Ondrej Slaby $\mathbb{1}^{1,2}$

\begin{abstract}
Growing evidence suggests that microRNAs are involved in the development and progression of colorectal cancer (CRC). In the present study, deregulation and functioning of tumor-suppressive miR-215-5p was evaluated in CRC. In total, 448 tumor tissues and 325 paired adjacent healthy tissues collected from Czech and Spain cohorts of CRC patients have been used for miR-215-5p expression analyses. A series of in vitro experiments have been performed using transient transfection of miR-215-5p mimics into four CRC cell lines to identify specific cellular processes affected by miR-215-5p. Further, the effects of miR-215-5p on tumor growth were evaluated in vivo using NSG mice and stable cell line overexpressing miR-215-5p. Target mRNAs of miR-215-5p were tested using luciferase assay and western blot analyses. We found that miR-215-5p is significantly downregulated in tumor tissues compared with non-tumor adjacent tissues and its decreased levels correlate with the presence of lymph node metastases, tumor stage, and shorter overall survival in CRC patients. Overexpression of miR-215-5p significantly reduced proliferation, clonogenicity, and migration of CRC cells, lead to cell cycle arrest in G2/M phase and p53-dependent induction of apoptosis. The ability of miR-215-5p to inhibit tumor growth was confirmed in vivo. Finally, we confirmed epiregulin and HOXB9 to be the direct targets of miR-215-5p. As epiregulin is EGFR ligand and HOXB9 is its transcriptional inducer, we suggest that the main molecular link between miR-215-5p and CRC cells phenotypes presents the EGFR signaling pathway, which is one of the canonical pathogenic pathways in CRC.
\end{abstract}

\section{Introduction}

Colorectal cancer (CRC) is the third most common cancer worldwide and the fourth leading cause of cancer related deaths. Despite the fact that the incidence and mortality rates have been steadily declining, $>50 \%$ of all patients with CRC will die of the disease ${ }^{1}$. In recent years,

\footnotetext{
Correspondence: Ondrej Slaby (on.slaby@gmail.com)

${ }^{1}$ Central European Institute of Technology, Masaryk University, Brno, Czech Republic

${ }^{2}$ Department of Comprehensive Cancer Care, Masaryk Memorial Cancer Institute, Faculty of Medicine, Masaryk University, Brno, Czech Republic Full list of author information is available at the end of the article
}

many different classes of non-coding RNAs have been identified as key regulators of various cellular processes including cell proliferation, differentiation, apoptosis or migration $^{2-5}$. MicroRNAs (miRNAs) are short singlestranded non-coding RNAs that post-transcriptionally regulate gene expression by binding to $3^{\prime}$ untranslated regions of target mRNAs ${ }^{6}$. Many studies have shown they can act as both oncogenes and tumor suppressors and their deregulation has been associated with the initiation and progression of a wide range of human diseases, including cancer ${ }^{7}, 8$. In addition, association between 
miRNA expression, prognosis and therapy response prediction was repeatedly described ${ }^{9,} 10$.

Over the past decade, several miRNAs with deregulated expression in CRC have been identified, including miR$215-5 p^{11-15}$. We focus on miR-215-5p as we identified this miRNA to be downregulated in colorectal tumor tissue in our previous work ${ }^{11}$, where it indicated also promising tumor-suppressive features in preliminary in vitro functional screen ${ }^{11}$. In general, this miRNA is supposed to function as a tumor suppressor and its levels are often downregulated in tumor tissues. However, its role in CRC pathogenesis has not been fully elucidated yet. In 2008, miR-215 has been shown to act as an effector as well as regulator of $\mathrm{p} 53^{13}$. Further, denticleless protein homo$\log ^{14}$ and thymidylate synthase ${ }^{15}$ were confirmed to be the miR-215-5p targets. Low expression levels of miR-215-5p were associated with resistance to 5-fluorouracilcontaining adjuvant chemotherapy ${ }^{16}$. Finally, the deregulation of this miRNA is supposed to be a very early event, which is not dependent on the mechanism of initiation of transformation, suggesting that miR-215-5p is likely to regulate critical signaling pathways that are crucial for early transformation of colonic epithelial cells ${ }^{12}$.

In this study, we have determined expression levels of miR-215-5p in two large independent cohorts of CRC patients to confirm its downregulation in tumor tissue and prognostic potential. To further discover the role of miR-215-5p in CRC pathogenesis, we have performed deep in vitro analyses with the aim to describe the most significantly affected CRC cells phenotypes and identify mRNA targets and the key signaling pathways affected by miR-215-5p. The role of miR-215-5p in regulation of tumor growth was evaluated also in vivo using mouse model.

\section{Results}

MiR-215-5p is downregulated in CRC tissues and its low levels correlate with aggressive disease

It was confirmed that the expression of miR-215-5p is significantly downregulated in tumor tissue compared with adjacent mucosa $(P<0.0001$; Fig. 1a) in case of Czech cohort (Table 1). In addition, the levels of miR-215$5 \mathrm{p}$ decreased progressively with advanced clinical stages $(P<0.0001$; Fig. 1c) and low expression was associated with lymph nodes positivity $(P<0.0001$; Supplementary Fig. S1A). Further, significantly downregulated levels of miR-215-5p were found not only in primary tumors, but also in corresponding liver metastases $(P<0.0001$; Supplementary Fig. S1B). Survival analyses proved that patients with low levels of miR-215-5p have significantly shorter overall survival (OS) $(P=0.0024$; cut-off 0.02393 ; Fig. 1e) compared with patients with higher expression levels.
To further validate these observations, an independent cohort from Spain was included in the study (Table 1). As in the Czech cohort, the expression of miR-215-5p was significantly downregulated in tumor tissues $(P<0.0001$; Fig. 1b) and its low levels were associated with advanced clinical stage $(P=0.0185$; Fig. $1 \mathrm{~d})$, but not with the lymph node positivity (Table 1). Again, the low levels of miR215-5p were associated with shorter OS and worse prognosis of CRC patients $(P=0.0111$; cut-off 0.2139 ; Fig. 1f).

\section{MiR-215-5p expression levels in CRC cells}

MiR-215-5p expression levels in CRC cells used in our study was performed by use of calibration curve (Supplementary Fig. S7A) and absolute quantification. The number of miR-215-5p copies varied among CRC cells (Supplementary Fig. S7B). HCT-116 $6^{+/+}$HCT- $116^{-/-}$, DLD-1 and HT-29 were characteristic with very low number of miR-215-5p copies ranging from 1351 to 3639 copies per $100 \mathrm{ng}$ of total RNA purified from CRC cells. On the contrary, the only CRC cells indicating multiple time higher levels were $\mathrm{CaCo} 2$ cells with number of copies 98962 per $100 \mathrm{ng}$ of total RNA. Based on this results, HCT- $116^{+/+}$, HCT-116 ${ }^{-/-}$, DLD-1 and HT-29 cells were used as models for miR-215-5p substitution and $\mathrm{CaCo} 2$ for miR-215-5p silencing.

\section{MiR-215-5p inhibits proliferation, viability and colony formation of CRC cells}

By transfection of miR-215-5p, mimic reached significant increase of miR-215-5p levels in all studied cell lines, which was stable from 24 to $96 \mathrm{~h}$. Expression levels of miR-215-5p in cells transfected with miR-215-5p mimic were 8000-10000 times higher when compared with mock-transfected control cells. Cell counting demonstrated cell proliferation to be inhibited by ectopic expression of miR-215-5p, with the best inhibition effect being observed $96 \mathrm{~h}$ after transfection $(P<0.001$ for HCT-116 ${ }^{+/+}$and HT-29; $P<0.01$ for DLD-1 and HCT$116^{-/-}$; Figs. 2a, b, Supplementary Figs. S2A, B). Parallel to cell counting, MTT (3-(4,5-dimetylthiazol-2-yl)-2,5difenyltetrazolium bromid) assay was performed to assess the effect of miR-215-5p on cell viability. Similarly to the previous results, the viability of CRC cells was significantly reduced $96 \mathrm{~h}$ after transfection $(P<0.001$ for HCT-116 $6^{+/+}$and HT-29; $P<0.01$ for DLD-1; $P<0.05$ for HCT $-116^{-1-}$ ). To determine whether the alterations in cell proliferation and viability were the result of cell cycle regulation, flow cytometry was used. Ninety-six hours after transfection, miR-215-5p decreased the proportion of HCT-116 $6^{+/+}$(Fig. 2c), HCT-116 ${ }^{-/-}$(Supplementary Fig. S2C) and DLD-1 (Fig. 2d) cells in the G0/G1-phase and increased the proportion of the cells in S-phase and G2/M-phase compared with those transfected with 


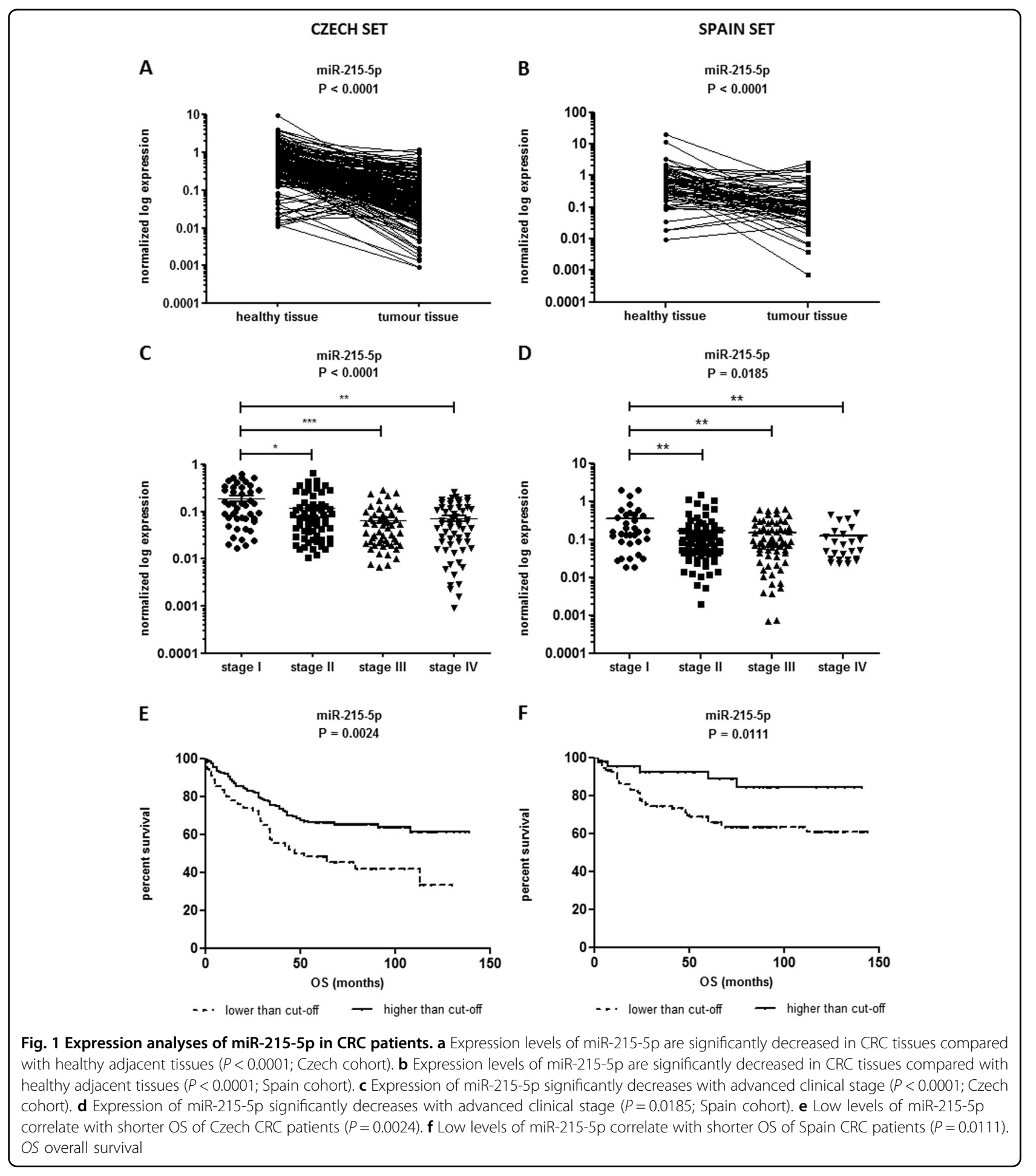

control oligonucleotides. In case of HT-29 cells, only the arrest in G2/M-phase was observed (Supplementary Fig. S2D). To find out whether the inhibition of growth induced by miR-215-5p was anchorage independent, the cells were seeded on soft agar $24 \mathrm{~h}$ post-transfection. After
14 days, HCT- $116^{+/+}$and DLD-1 cells transfected with miR-215-5p mimics formed significantly fewer colonies than cells transfected with control oligonucleotide $(P<$ 0.001; Figs. 2e, f). 
Table 1 Correlation of miR-215-5p expression with clinical-pathological features of CRC patients

\begin{tabular}{|c|c|c|c|c|}
\hline & $n(\%)$ & miR-215-5p Czech cohort median (25-75\%) & $n(\%)$ & miR-215-5p Spain cohort median (25-75\%) \\
\hline \multicolumn{5}{|l|}{ Age } \\
\hline Median (range) & $66(18-92)$ & NA & $73(36-91)$ & NA \\
\hline \multicolumn{5}{|l|}{ Sex } \\
\hline Male & $139(55)$ & $0.07(0.02-0.15)$ & $107(55)$ & $0.07(0.03-0.17)$ \\
\hline Female & $113(45)$ & $0.07(0.03-0.014)$ & $89(45)$ & $0.10(0.04-0.20)$ \\
\hline$P$-value & & 0.9673 & & 0.3508 \\
\hline \multicolumn{5}{|l|}{ Tumor vs. mucosa } \\
\hline Normal mucosa & 252 & $0.51(0.33-0.87)$ & 73 & $0.58(0.27-1.19)$ \\
\hline Colorectal tumor & 252 & $0.07(0.03-0.14)$ & 196 & $0.11(0.05-0.25)$ \\
\hline$P$-value & & $<0.0001$ & & $<0.0001$ \\
\hline Clinical stage & $n=252$ & & $n=192$ & \\
\hline । & $43(17)$ & $0.14(0.07-0.31)$ & $23(12)$ & $0.15(0.09-0.43)$ \\
\hline$\|$ & $78(31)$ & $0.07(0.03-0.13)$ & $82(43)$ & $0.08(0.05-0.16)$ \\
\hline III & $60(24)$ & $0.04(0.02-0.08)$ & $64(33)$ & $0.08(0.04-0.20)$ \\
\hline IV & $71(28)$ & $0.05(0.02-0.12)$ & $23(12)$ & $0.05(0.03-0.15)$ \\
\hline$P$-value & & $<0.0001$ & & 0.0185 \\
\hline pT category & $n=252$ & & $n=193$ & \\
\hline pT1 & $4(2)$ & $0.30(0.08-0.60)$ & $10(5)$ & $0.04(0.03-0.08)$ \\
\hline pT2 & $51(20)$ & $0.09(0.05-0.26)$ & $18(9)$ & $0.05(0.01-0.20)$ \\
\hline pT3 & $172(68)$ & $0.06(0.02-0.13)$ & $124(65)$ & $0.09(0.04-0.16)$ \\
\hline pT4 & $25(10)$ & $0.04(0.02-0.09)$ & $41(21)$ & $0.10(0.05-0.20)$ \\
\hline$P$-value & & 0.0018 & & 0.2515 \\
\hline Lymph nodes & $n=252$ & & $n=192$ & \\
\hline Negative & $135(54)$ & $0.09(0.04-0.21)$ & $110(57)$ & $0.09(0.05-0.19)$ \\
\hline Positive & $117(46)$ & $0.04(0.02-0.10)$ & $82(43)$ & $0.08(0.04-0.20)$ \\
\hline$P$-value & & $<0.0001$ & & 0.4397 \\
\hline Distant metastases & $n=252$ & & $n=193$ & \\
\hline No & $181(72)$ & $0.07(0.03-0.14)$ & $170(88)$ & $0.08(0.04-0.15)$ \\
\hline Yes & $71(28)$ & $0.06(0.02-0.15)$ & $23(12)$ & $0.14(0.03-0.30)$ \\
\hline$P$-value & & 0.2780 & & 0.3306 \\
\hline Grading & $n=252$ & & $n=196$ & \\
\hline G1 & $67(27)$ & $0.07(0.03-0.26)$ & $144(73)$ & $0.09(0.04-0.20)$ \\
\hline G2 & $132(52)$ & $0.07(0.03-0.12)$ & $47(24)$ & $0.08(0.04-0.14)$ \\
\hline G3 & $53(21)$ & $0.04(0.02-0.16)$ & $5(3)$ & $0.13(0.02-0.31)$ \\
\hline$P$-value & & 0.1771 & & 0.4259 \\
\hline Tumor location & $n=252$ & & $n=194$ & \\
\hline Proximal colon & $100(40)$ & $0.07(0.03-0.18)$ & $104(54)$ & $0.09(0.04-0.19)$ \\
\hline Distal colon & $152(60)$ & $0.07(0.02-0.13)$ & $90(46)$ & $0.07(0.03-0.17)$ \\
\hline$P$-value & & 0.4787 & & 0.1817 \\
\hline
\end{tabular}

The $P$-values in bold are statistically significant NA not applicable 


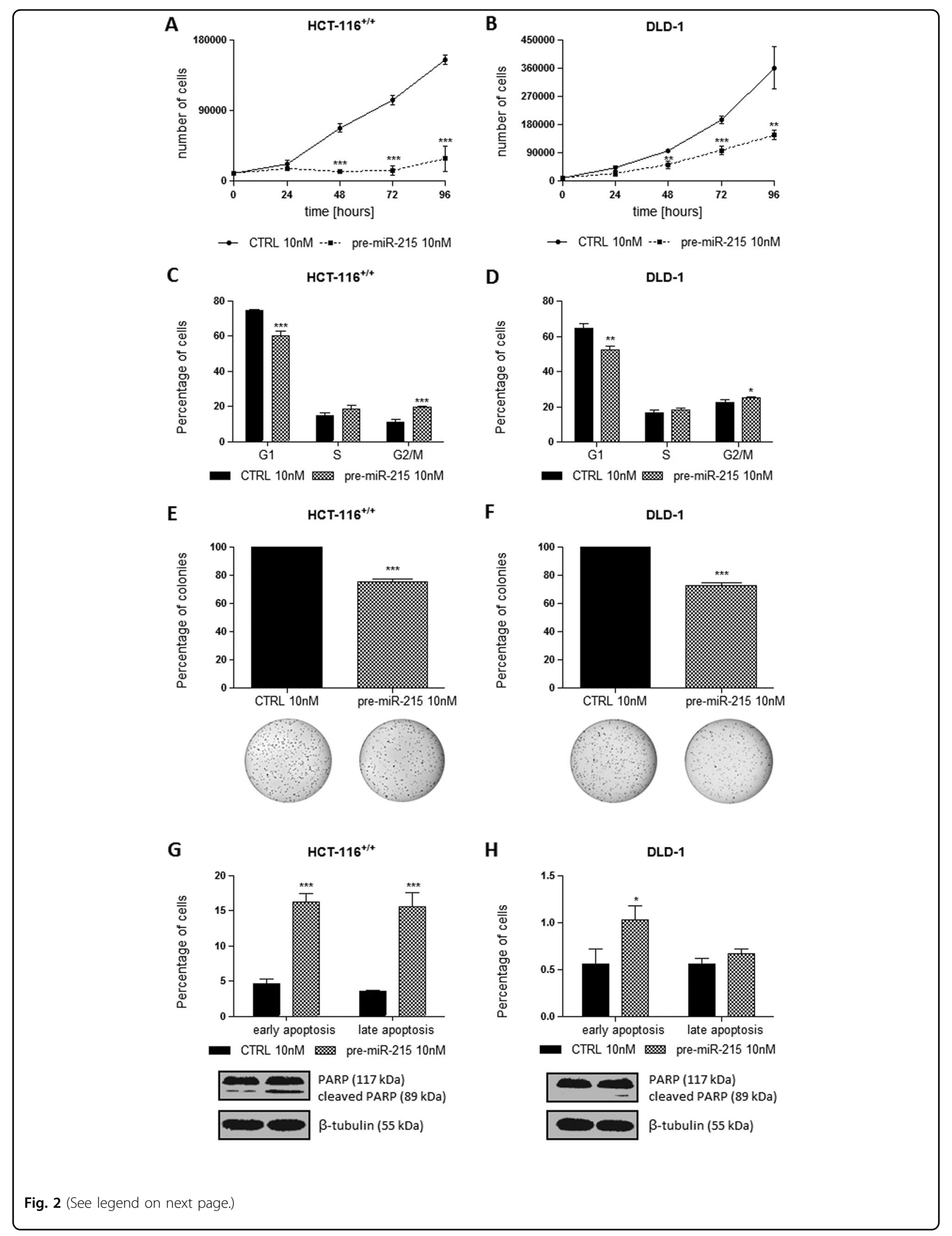


MiR-215-5p induces apoptosis of CRC cells in a wild-type p53-dependent manner

Transfection of miR-215-5p into HCT-116 ${ }^{+/+}$cells (wtp53) significantly increased the number of apoptotic cells by at least threefold ( $P<0.001$; Fig. $2 \mathrm{~g})$. Further, the levels of cleaved PARP were increased subsequent to transfection with miR-215-5p (Fig. 2g). Importantly, transfection of miR-215-5p mimics into HCT-116 ${ }^{-1-}$ (p53-null), DLD-1 (mut-p53) and HT-29 (mut-p53) cells did not lead to increased apoptosis rates and elevated levels of cleaved PARP (Fig. 2h, Supplementary Figs. S2E-F).

\section{MiR-215-5p inhibits migration of CRC cells}

According to the results of scratch wound assay, transfection of miR-215-5p mimics led to a significant inhibition of cell migration $(P<0.001$ for DLD-1, HCT$116^{+/+}$and HCT-116 $6^{-/-} ; P<0.05$ for HT-29; Figs. 3a, b and Supplementary Figs. S3A, B). In addition, transwell migration assay confirmed significantly reduced migration of cells overexpressing miR-215-5p. The inhibition rate was $57 \pm 16 \%, 37 \pm 8 \%$, and $50 \pm 17 \%$, respectively, in HCT $-116^{+/+}$(Fig. 3c), DLD-1 (Fig. 3d), and HCT-116 $6^{-1-}$ cells transfected with miR-215-5p mimics compared with control group.

\section{EREG and HOXB9 are direct targets of miR-215-5p}

To better understand the role of miR-215-5p in CRC pathogenesis, the TargetScan ${ }^{17}$, DIANA-micro ${ }^{18}$, RNAhybrid $^{19}$, miRanda ${ }^{20}$ and RNA22 ${ }^{21}$ databases were searched for the predicted targets of miR-215-5p associated with cell proliferation and migration. Among the target genes that were considered to be the most likely involved in these processes, epiregulin (EREG) ${ }^{22}$ and HOXB $9^{23}$ have been chosen for further analyses. It was found that miR-215-5p transfection leads to the significant decrease in mRNA levels of both genes of interest (Figs. 4a-d). Subsequently, the luciferase reporter assay was utilized to confirm direct interaction between miR215-5p and 3'-UTR of EREG and HOXB9. It was shown that miR-215-5p suppressed $62 \pm 6 \%$ of reporter activity of the pEZX-MT05-EREG reporter compared with the control oligonucleotide, whereas the pEZX-MT05-ctrl vector was resistant to the inhibition $(P<0.001$; Fig. 4e). Similarly, the reporter activity of the pLSG-RenSPHOXB9 reporter was suppressed by $48 \pm 3 \%$ after transfection of miR-215-5p compared with control cells, whereas the pLSG-RenSP-ctrl vector was resistant to the inhibition $(P<0.001$; Fig. 4f $)$. In addition, western blot analyses proved that overexpression of miR-215-5p suppresses the expression of both proteins $48 \mathrm{~h}$ after transfection (Fig. 4g). To further support these data, depletion of EREG and HOXB9 using small interfering RNA (siRNA)-mediated knockdown was performed (Supplementary Figs. S4A-D). It was shown that downregulation of these two proteins leads to the significant decrease in proliferation of HCT-116 ${ }^{+/+}(P<0.001$ in case of EREG, $P<0.01$ in case of HOXB9; Supplementary Fig. S5A), HCT-116 ${ }^{-1-}(P<0.01$ in case of EREG; Supplementary Fig. S5B) and DLD-1 $(P<0.01$ in case of EREG, $P<0.05$ in case of HOXB9; Supplementary Fig. S5C) cells $96 \mathrm{~h}$ after transfection. By use of scratch wound assay, we were not able to prove any significant effects of EREG and HOXB9 silencing on migratory capacity of studied cells $(P>0.05)$. Finally, the levels of EREG and HOXB9 were examined in the matched tumor and non-tumor tissues of CRC patients. It was shown that the expression of EREG and HOXB9 is significantly increased in tumor tissues compared with healthy tissues $(P<0.01$ for EREG, $P<0.001$ for HOXB9; Fig. 4h).

\section{MiR-215 induces increase in E-cadherin expression}

When we compared the expression levels of EMT markers (E-cadherin, vimentin, ZEB1, ZEB2) in HCT$116^{+/+}$-miR-215-5p cells and HCT-116 ${ }^{+/+}$-control cells, we observed significantly higher levels of E-cadherin $(P=$ 0.0164, Supplementary Fig. S6) in miR-215-5p-positive cells. There was no difference in vimentin and ZEB1 expression levels between studied cell lines. ZEB2 was not detectable in both cell lines.

\section{MiR-215-5p silencing facilitate proliferation of CRC cells and induce expression of EREG and HOXB9}

We successfully silenced miR-215-5p expression in $\mathrm{CaCo} 2$ cells to $16 \%$ of its expression levels in control $\mathrm{CaCo} 2$ cells transfected with anti-miRNA control oligonucleotide (Supplementary Fig. S8A). Silencing of miR215-5p by use of anti-miR-215 in CaCo2 cells led to the increase in expression levels of miR-215-5p targets EREG and HOXB9 after $48 \mathrm{~h}$ (Supplementary Fig. S8B). Finally, decreased levels of miR-215-5p facilitated proliferation of

\section{(see figure on previous page)}

Fig. 2 Effects of miR-215-5p overexpression on HCT-116 $6^{+/+}$and DLD-1 cells. a miR-215-5p significantly inhibits the proliferation of HCT-116 $6^{+/+}$ cells. $\mathbf{b}$ miR-215-5p significantly inhibits the proliferation of DLD-1 cells. c miR-215-5p significantly reduce the clonogenicity of HCT-116 ${ }^{+/+}$cells. $\mathbf{d}$ miR-215-5p significantly reduce the clonogenicity of DLD-1 cells. e Overexpression of miR-215-5p in HCT-116 $6^{+/+}$cells leads to a cell cycle arrest in G2/ M phase. $\mathbf{f}$ Overexpression of miR-215-5p in DLD-1 cells leads to a cell cycle arrest in G2/M phase. $\mathbf{g}$ miR-215-5p increases the apoptosis of HCT-116 ${ }^{+1}$ ${ }^{+}$cells in p53-dependent manner. $\mathbf{h}$ miR-215-5p increases the early apoptosis of DLD-1 cells. ${ }^{*} P<0.05,{ }^{*} P<0.01,{ }^{* * *} P<0.001$, CTRL control cells 


\section{A}

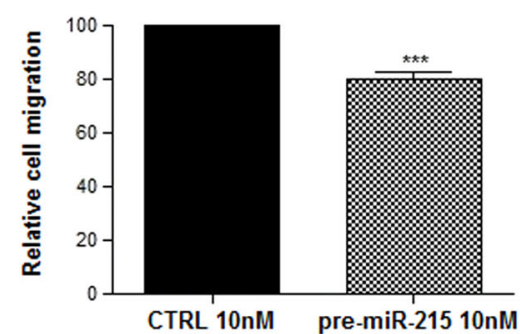

$\mathrm{Oh}$

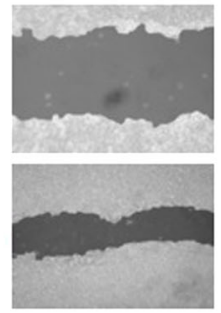

C
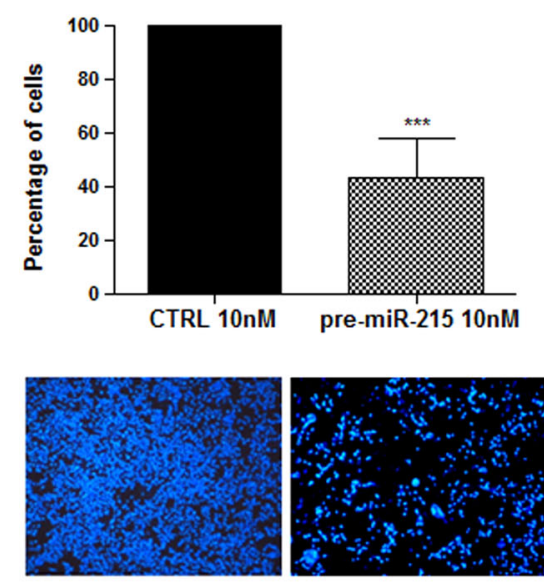

B

DLD-1

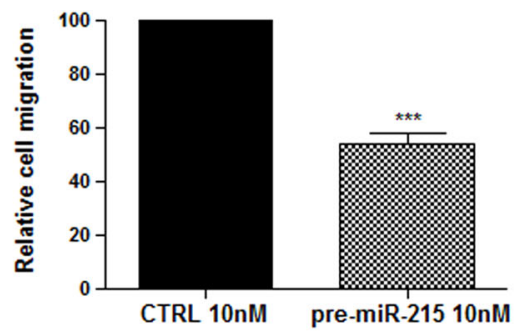

$\mathrm{Oh}$

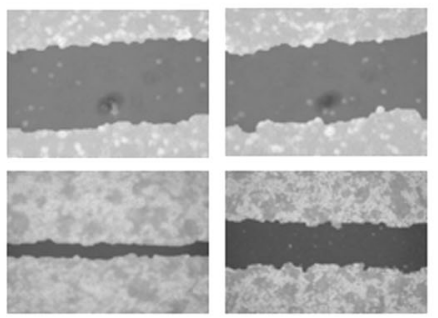

D DLD-1


Fig. 3 Effects of miR-215-5p overexpression on migration of HCT-116 ${ }^{+/+}$and DLD-1 cells. a miR-215-5p significantly reduce the migration of $\mathrm{HCT}-116^{+/+}$cells (transwell migration assay). b miR-215-5p significantly reduce the migration of DLD-1 cells (transwell migration assay). $\mathbf{c}$ miR-215-5p significantly reduce the migration of $\mathrm{HCT}-116^{+/+}$cells (scratch wound assay). $\mathbf{d}$ miR-215-5p significantly reduce the migration of DLD-1 cells (scratch wound assay). ${ }^{* *} P<0.01,{ }^{* * *} P<0.001, C T R L$ control cells

CaCo2 cells, which was significant at first $(P=0.03)$ and second day $(P=0.02)$ post-transfection (Supplementary Fig. S8C). We have not observed any significant effects of miR-215-5p silencing on migratory capacity of $\mathrm{CaCo} 2$ cells by use scratch wound-healing assay $(P>0.05)$.

\section{MiR-215-5p overexpression suppresses tumor growth in vivo}

To evaluate how miR-215-5p overexpression affects tumor growth in vivo, subcutaneous tumors were generated in NSG mice using HCT-116 $6^{+/+}$-miR-215-5p cells and HCT- $116^{+/+}$-control cells. It was confirmed that HCT $-116^{+/+}$-control tumors grow significantly faster than the HCT-116 ${ }^{+/+}$-miR-215-5p tumors (Figs. 5a-c).
Importantly, reverse transcriptase-quantitative PCR (RTqPCR) analysis showed that the expression of miR-215-5p is still upregulated in HCT-116 ${ }^{+/+}$-miR-215-5p tumors compared with control tumors (Fig. 5d) 25 days from the beginning of the experiment.

\section{Discussion}

Growing evidence suggests that miRNAs are involved in the development and progression of different types of human cancers $^{24-26}$. In 2008, miR-215-5p was first described as a tumor suppressor in $\mathrm{CRC}^{13}$. Since then, several other authors studied prognostic and predictive value of miR-215-5 $\mathrm{p}^{14-16}$; however, its detail functioning in the pathogenesis of the disease has not been clarified 


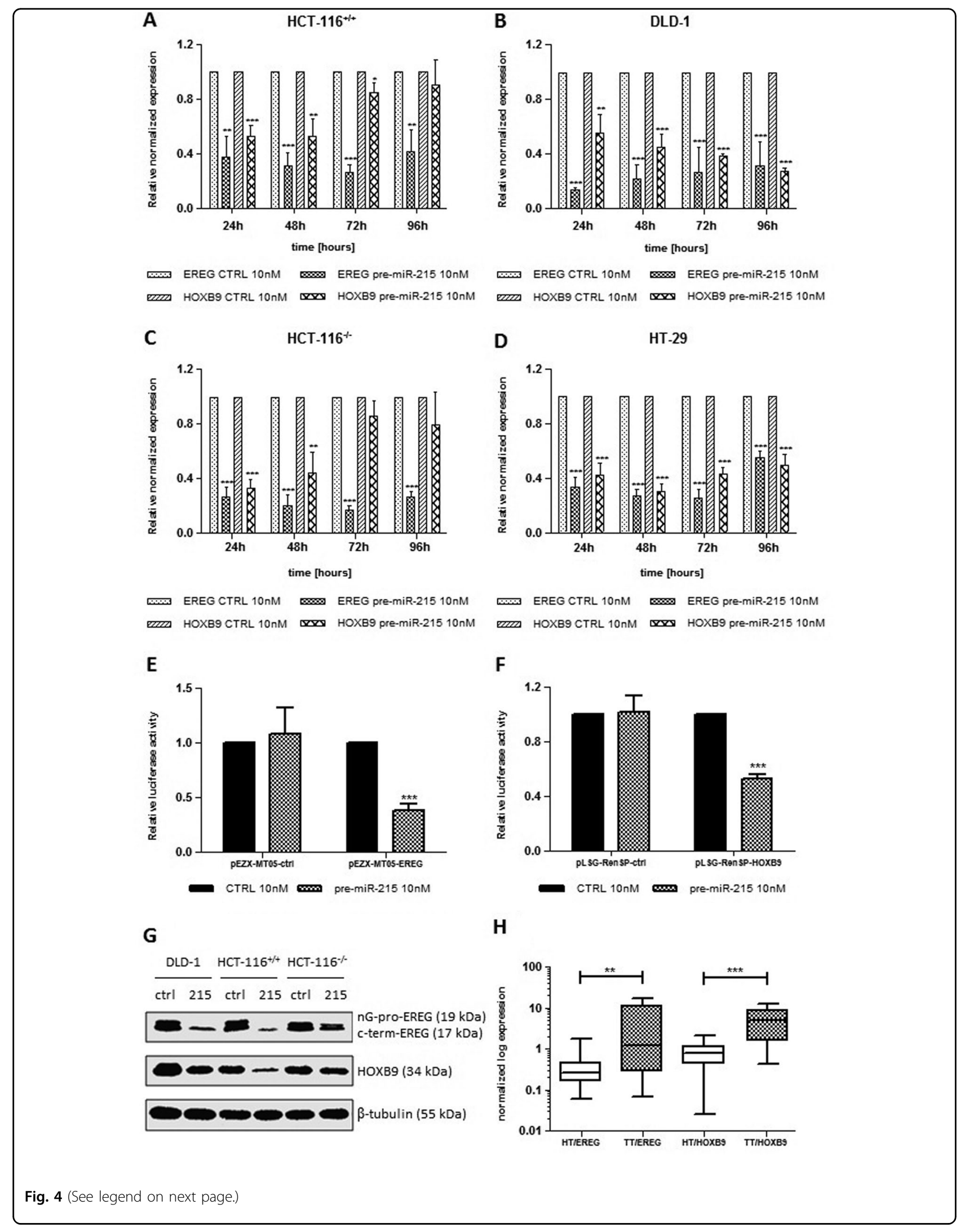


yet. Thus, we have analyzed not only the diagnostic and prognostic potential of miR-215-5p, but we also aimed to identify its target genes and describe its involvement in CRC cells phenotypes and particular signaling pathways.

We have confirmed that the expression of miR-215-5p is significantly downregulated in tumor tissues compared with paired healthy tissues and its reduced levels correlate with higher clinical stage, presence of lymph node metastases and shorter OS. These results are in agreement with former studies ${ }^{27-30}$.

To identify specific cellular processes influenced by miR-215-5p, series of in vitro experiments have been performed. The results proved that higher levels of miR215-5p significantly reduce metabolic activity and proliferation of CRC cell lines. The opposite effects were observed when miR-215-5p silencing approach was used in $\mathrm{CaCo} 2$ cells, where decreased levels of miR-215-5p led to enhanced cellular proliferation. Similarly, the in vivo experiments confirmed significantly slower growth of tumors stably expressing this miRNA. In compliance with previous studies ${ }^{13,14}$, the inhibitory effect was more profound in cells containing wild-type p53 (HCT-116 ${ }^{+/+}$) compared with p53-mutant (DLD-1, HT-29) or p53-null $\left(\mathrm{HCT}-116^{-1-}\right)$ cells. On the other hand, miR-215-5p significantly reduced cell proliferation even in the absence of p53; thus it seems that this miRNA slows down the proliferation not only through the cell cycle arrest, but also by affecting another signaling pathways independent of p53 function. Concerning the cell cycle, transfection of miR-215-5p lead to the significant arrest in G2/M phase. These results again support the hypothesis of other proteins than p53 being involved in reduced proliferation. Georges et al. confirmed denticleless protein homolog to be a direct target of miR-215-5p that interacts with DDB1-CUL4 and MDM2-p53 ligase complexes and influences the stability of p53 and its target p $21^{31,32}$. Similarly, Boni et al. identified thymidylate synthase as another target of miR-215-5p that was suggested to be a predictive biomarker for 5-FU response in CRC. To find out whether the inhibition of growth induced by miR215-5p was anchorage independent, the clonogenic assay was performed ${ }^{15}$. It was revealed that number of colonies is significantly lower in case of HCT- $116^{+/+}$and DLD-1 cells transfected with miR-215-5p mimics, but not in
HCT $-116^{-1-}$ and HT-29 cells indicating that this miRNA could affect the cell clone formation by mechanisms that are at least in part dependent on p53 functionality. Interestingly, although carrying out the above experiments significant morphologic changes in HCT $-116^{+/+}$ cells transfected with miR-215-5p, such as round shape and plate surface detachment, have been repeatedly observed. These changes may have many reasons including reduced expression of adhesion molecules, loss of cell polarity, dysfunctional cytoskeleton or cell apoptosis $^{33}$. Georges et al. identified discs large homolog 5 as an important target of miR-215-5p $\mathrm{p}^{31}$. It was shown that this protein can interfere with cell adhesion through the reduction of cadherin transport to the cell surface and it is proposed to function in the maintenance of epithelial cell structure $^{34}$. Concerning the effect of miR-215-5p on cell apoptosis, it was assessed that overexpression of this miRNA leads to the significant increase of apoptotic rate in case of HCT-116 $6^{+/+}$cells. Although the exact mechanism of action is not known, it was proved that this outcome is strongly dependent on the presence of wt-p53 and could be related to morphologic changes described earlier. To date, no target genes of miR-215-5p associated with cell apoptosis have been identified in CRC. Nevertheless, X-chromosome-linked inhibitor of apoptosis (XIAP) was found to be regulated by this miRNA in ovarian $^{35}$ and non-small cell lung cancer ${ }^{36}$.

Further, we observed that higher levels of miR-215-5p lead to a significant inhibition of cell migration. Interestingly, the highest effect was determined in case of DLD-1 cell line indicating the independence of p53 status. In 2011, White et al. identified ZEB2 as a direct target of miR-215-5p in renal cell carcinoma ${ }^{37}$. These results were further confirmed using non-small cell lung cancer ${ }^{38}$ and pancreatic cancer ${ }^{39}$ cell lines. Using the metastatic gene profiling assay, several other genes involved in the degradation of extracellular matrix or cell adhesion, such as MMP7/13 or CDH1/6/11, have been described to be affected by increased miR-215-5p expression in renal cell carcinoma ${ }^{37}$; however, these targets need to be further validated in CRC.

As our observations proved significant effects of miR215-5p on cell proliferation and migration, several databases have been searched for the potential targets of miR-

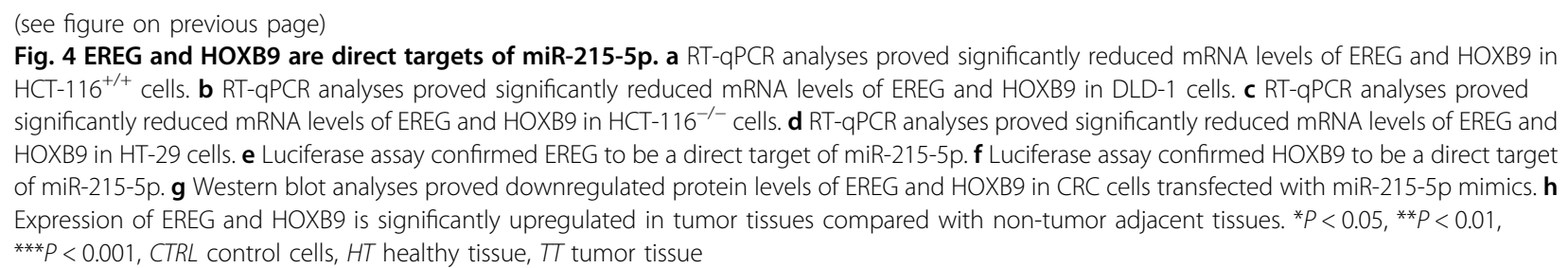


A

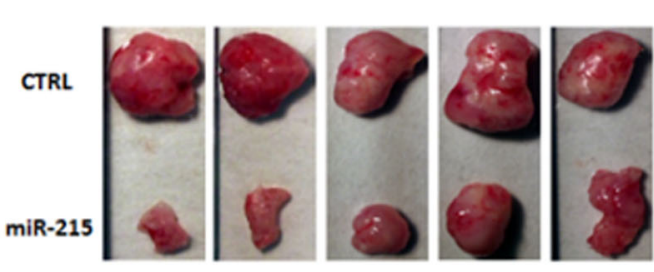

C

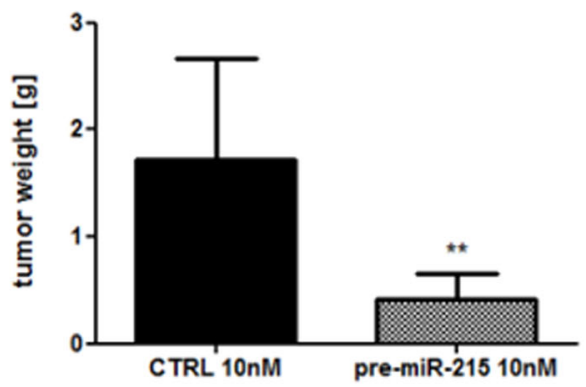

B

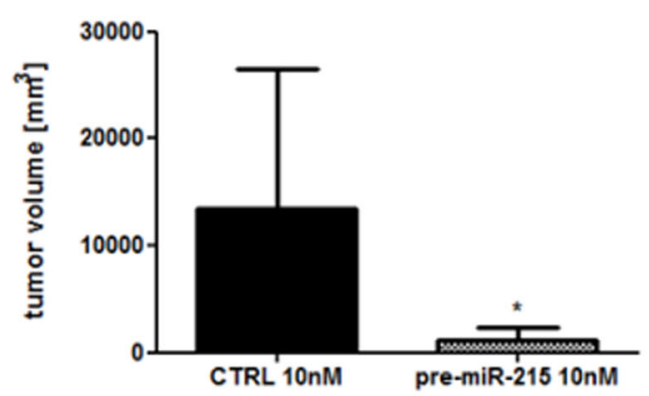

D

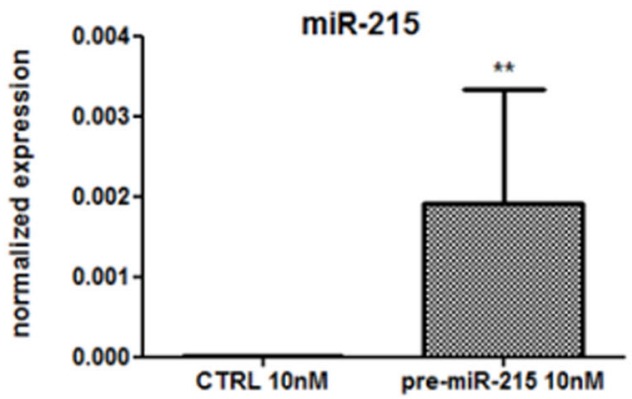

E

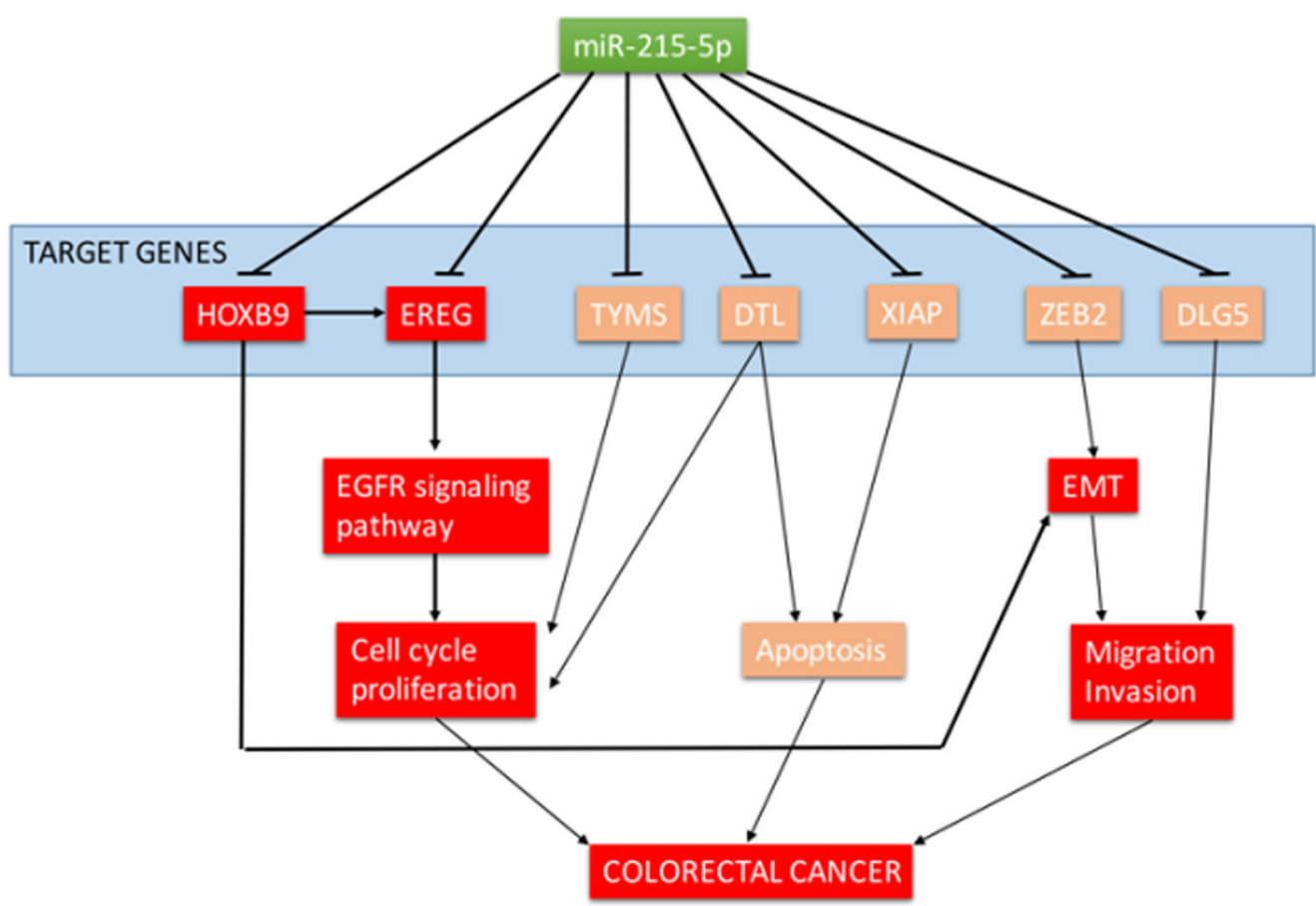

Fig. 5 Effects of miR-215-5p overexpression on tumor growth in vivo and its involvement in CRC pathogenesis. a Subcutaneously injected HCT-1 $16^{+/+}$-miR-215-5p cells formed significantly smaller tumors compared with HCT-116 $6^{+/+}$-control cells 25 days after application into NSG mice $(n=5)$. b Volume of in vivo formed tumors was significantly smaller in case of HCT-116 $6^{+/+}$-miR-215-5p cells compared with HCT-116 ${ }^{+/+}$-control cells. $\mathbf{c}$ Weight of in vivo formed tumors was significantly smaller in case of HCT-116 $6^{+/+}$-miR-215-5p cells compared with HCT-116 $6^{+/+}$-control cells. $\mathbf{d}$ Expression levels of miR-215-5p were significantly increased in HCT-116 $6^{+/+}$-miR-215-5p tumors compared with HCT-116 $6^{+/+}$-control tumors 25 days after initiation of the experiment. e Involvement of miR-215-5p in CRC pathogenesis - direct targets of miR-215-5p described first in this study are in red squares, direct targets of miR-215-5p described in previous studies are in brown. ${ }^{*} P<0.05,{ }^{* *} P<0.01$, CTRL control cells, EMT

epithelial-mesenchymal transition, XIAP X-chromosome-linked inhibitor of apoptosis, ZEB2 zinc-finger E-box-binding homeobox 2, DTL denticleless protein homolog, DLG5 discs large homolog 5, TYMS thymidylate synthase, HOXB9 homeobox protein HoxB9, EREG epiregulin 
215-5p associated with these processes. From the predicted genes, EREG and HOXB9 have been chosen for further validation. The performed analyses proved these two proteins to be the direct targets of miR-215-5p. Moreover, their expression was significantly upregulated in tumor tissue compared with adjacent healthy tissue. EREG is a member of the epidermal growth factor family that functions as a ligand of EGFR, which is commonly overexpressed in CRC and present one of its main molecular features ${ }^{38}$. HOXB9 is an important transcription factor contributing to solid tumor invasion and metastasis and its overexpression is associated with poor prognosis $^{40,41}$. Interestingly, it was found that EREG promoter contains the HOX-binding site and is a direct transcriptional target of $\mathrm{HOXB} 9^{42}$. To date, no previous study has confirmed EREG and HOXB9 to be direct targets of miR-215-5p. However, Wu et al. identified HOXB9 as a direct target of miR-192, a miRNA from the same family and with a high homology to miR-215-5p ${ }^{43}$. The most prominent regulatory effect of miR-215-1p on HOXB9 was observed under p53-wild-type conditions, in cell line HCT- $116^{+/+}$, which could be partly explained by the fact that miR-192 ${ }^{13,44}$ and miR- $215^{44}$ have been shown to be p53-responsive miRNAs. As miR-215-5p has an ability to regulate EGFR ligand EREG and its transcriptional inducer HOXB9, we suggest that the main molecular link between miR-215-5p and CRC cells phenotypes presents the EGFR signaling pathway, which is one of the canonical pathogenic pathways in CRC (Fig. 5e).

In conclusion, we have confirmed a diagnostic and prognostic potential of miR-215-5p in CRC patients in two independent cohorts of patients. In addition, we have proved the tumor-suppressive character of miR-215-5p resulting in reduced proliferation, formation of new colonies, and migration and increased apoptosis. These results correspond with the fact that one miRNA has the ability to regulate several target genes involved in different signaling pathways. Although some of these effects were dependent on the presence of wt-p53, miR-215-5p was also able to slow down the tumor growth independently of this protein. Importantly, two genes-EREG and HOXB9 - that are functionally linked to EGFR signaling and are known to be involved in cell proliferation, migration and disease progression have been validated as direct targets of this miRNA. Thus, we believe that miR-215-5p could serve as a potential therapeutic target in CRC.

\section{Materials and methods}

\section{Patients and tissue samples}

In total, 252 tumor tissue samples from patients with histopathologically verified CRC who had undergone surgery at Masaryk Memorial Cancer Institute (Brno, Czech Republic) from 2004 to 2013, as well as 252 paired adjacent non-tumor tissues were used for the determination of miR-215-5p expression levels. In addition, 17 samples of corresponding liver metastases obtained from patients with metastatic CRC were used in our study. Further, an independent set of tumor tissues from 196 patients who had undergone the surgery at Santa Lucía General University Hospital (HGUSL, Cartagena, Spain) from 2004 to 2015 , as well as 73 paired adjacent non-tumor tissues were involved in the study. All subjects enrolled in the study were of the same ethnicity (European descent) and did not receive any treatment prior to surgery. All patients were followed-up for tumor recurrence at regular intervals and survival time was calculated. Clinical and pathological characteristics were recorded and are summarized in Table 1. Written informed consent was obtained from all participants and the study has been approved by the local Ethical Boards in Masaryk Memorial Cancer Institute and Santa Lucía General University Hospital.

\section{Tissue samples preparation and miRNA isolation}

Tissue samples were homogenized (MM301, Retsch GmbH \& Co. KG, Germany) and total RNA enriched for small RNAs was isolated using mirVana miRNA Isolation Kit (Ambion, Austin, TX, USA) according to the manufacturer's instructions. Concentration and purity of RNA were determined spectrophotometrically by measuring its optical density (A260/280 > 2.0; A260/230 > 1.8) using a Nanodrop ND-1000 (Thermo Fisher Scientific, Waltham, MA, USA).

\section{Reverse transcription and RT-qPCR}

For miRNA expression analyses, complementary DNA (cDNA) was synthesized from 10 ng of total RNA using gene-specific primers (has-miR-215-5p; ID 000518, RNU48; ID 001006) according to the TaqMan MicroRNA Assay protocol (Applied Biosystems, Foster City, CA, USA) and real-time PCR was performed using TaqMan Universal PCR Master Mix, NoUmpErase UNG (Applied Biosystems) as described previously ${ }^{11}$. For quantification of the number of miR-215-5p copies in CRC cells used in our study, a dilution series of synthetic miRNA oligo (IDT, Coralville, IA, USA) were carried out in parallel with qRT-PCR of biological samples to generate an absolute standard curve. MiR-215-5p levels in CRC cells were expressed as number of copies per $100 \mathrm{ng}$ of total RNA purified from CRC cells. For the purposes of gene expression analyses, cDNA was synthesized using $1000 \mathrm{ng}$ of total RNA and the High-Capacity cDNA Reverse Transcription Kit (Applied Biosystems) according to the manufacturer's recommendations. Quantitative PCR was carried out using specific probes for EREG (Hs00914313_m1), HOXB9 (Hs00256886_m1), PMM1 (Hs00160195_m1), CDH1 (Hs01023895_m1), VIM 
(Hs00958111_m1), ZEB1 (Hs01566408_m1), ZEB2 (Hs00207691_m1) and GAPDH (glyceraldehyde-3-phosphate dehydrogenase, Hs02758991_g1) (Applied Biosystems). Real-time PCR was performed using the Applied Biosystems 7500 Sequence Detection System.

\section{Cell lines and cell culture}

In this study, four human colon carcinoma cell lines were used including HCT-116 $6^{+/+}\left(\mathrm{CCL}-247^{\mathrm{TM}}\right.$; wt-p53), DLD-1 (CCL-221 ${ }^{\mathrm{TM}}$; mut-p53), HT-29 (HTB-38 ${ }^{\mathrm{TM}}$; mutp53), $\mathrm{CaCo} 2\left(\mathrm{HTB}-37^{\mathrm{TM}}\right.$, mut-p53) and HCT- $116^{-/-}$ (p53-null derivative). The first four cell lines were obtained from American Type Culture Collection (ATCC), the HCT $-116^{-1-}$ cells were kindly provided by Dr Jiri Kohoutek (Veterinary research institute, Brno, Czech Republic) who gained them from Dr Bert Vogelstein $^{45}$. Cells were cultured in Dulbecco's modified Eagle's medium (DMEM) supplemented with $10 \%$ fetal bovine serum, $100 \mu \mathrm{g} \mathrm{ml}^{-1}$ penicilin, $100 \mu \mathrm{g} \mathrm{ml}^{-1}$ streptomycin, $0.1 \mathrm{mM}$ non-essential amino acids, $2 \mathrm{mM}$ L-glutamin, 1 $\mathrm{mM}$ sodium pyruvate (Invitrogen, Gibco, Carlsbad, CA, USA) in $5 \% \mathrm{CO}_{2}$ at $37^{\circ} \mathrm{C}$. All cell lines were regularly tested with MycoAlert (Lonza Group Ltd, Basel, Switzerland) to ensure the absence of mycoplasma contamination. Authentication of cell lines was done by comparing STR (short tandem repeat) sequences obtained from actual cell lines as determined by Generi Biotech (Hradec Kralove, Czech Republic) with data public available (ATCC, ECACC-European Collection of Authenticated Cell Cultures). Recent STR analysis has been performed within 6 months before the beginning or in the course of the experiments for all cell lines.

\section{Cell transfection}

All cell lines were transfected with $10 \mathrm{nM}$ hsa-miR-2155p mimic (MC10874) or miRNA Mimic, negative control \#1 (4464058) or $33 \mathrm{nM}$ hsa-miR-215-5p inhibitor (MH10874) or $33 \mathrm{nM}$ miRNA inhibitor, negative control (4464079) or $30 \mathrm{nM}$ siRNA-negative control (AM4635), siEREG (145900) and siHOXB9 (109525; all from Ambion) $24 \mathrm{~h}$ after seeding using Lipofectamine RNAiMAX transfection reagent (Invitrogen) according to the manufacturer's protocol. Transfection efficiency was evaluated by RT-qPCR.

\section{Cell proliferation and MTT assay}

Cells were seeded in triplicates in 10\% DMEM without antibiotics in 24-well plates $24 \mathrm{~h}$ before transfection and counted 24-96h after transfection. Cell viability was measured using the MTT assay (Sigma Aldrich, Saint Louis, MO, USA). The absorbance was measured on Multi-Detection Microplate Reader (BIO-TEK, Winooski, VT, USA).

\section{Colony-forming assay}

Colony-forming assay was performed using six-well plates pre-coated with $0.75 \%$ agarose as the bottom layer, whereas the top layer consisted of $0.35 \%$ agarose and tumor cells transfected with miR-215 mimics or control oligonucleotide. After 12-14 days, colonies were stained with crystal violet blue solution (Sigma-Aldrich) and scanned by GelCount (Oxford Optronix, Abingdon, UK). The data were analyzed using ImageJ software (Wayne Rasband, NIH, MD, USA).

\section{Cell cycle analysis and detection of apoptosis}

Cell cycle analysis and detection of apoptosis were performed using flow cytometry as described previously ${ }^{11}$. The cells were analyzed 72 and $96 \mathrm{~h}$ post-transfection.

\section{Scratch wound migration assay}

The migration of cells was analyzed using scratch wound migration assay. Cells were seeded on six-well plates and the cell monolayer was wounded $24 \mathrm{~h}$ after the transfection. The migration was measured at time 0 and $24 \mathrm{~h}$ post-wounding using a microscope Nikon Diaphod 300 INV $(10 \times)$ and camera Canon Power shot A95. Images were analyzed by the Tscratch software (CSElab, ETH Zurich, Switzerland).

\section{Transwell migration assay}

Transwell migration assay was performed using $8 \mu \mathrm{m}$ transwell inserts for 24-well plates (Costar, Corning Incorporated, Corning, NY, USA) and staining with Hoechst 33342 (Invitrogen). The migrated cells were counted using fluorescence microscope and ImageJ software (Wayne Rasband).

\section{Luciferase assay}

For luciferase reporter assay, MISSION 3'-UTR Lenti GoClone HOXB9 (HUTR10238) and appropriate negative control (HUTR001C) from SwitchGear Genomics (Carlsbad, CA, USA) were used and the viral particles were added at MOI (multiplicity of infection) $=2.5$. In case of EREG, $1 \mu \mathrm{g}$ of pEZX-MT05 vector containing UTR for EREG (HmiT004978) or appropriate control vector (CmiT000001-MT05) were transfected into DLD-1 cells using EndoFectin Plus Transfection Reagent (GeneCopoeia, Rockville, MD, USA). The luciferase activity was measured using the MISSION LightSwitch Luciferase Assay Reagent (Sigma Aldrich) or SecretePair Dual Luminescence assay kit (GeneCopoeia), respectively, using FLUOstar Omega Microplate reader (BMG Labtech, Ortenberg, Germany).

\section{Western blotting}

Cells were seeded in $60 \mathrm{~mm}$ plates and $48 \mathrm{~h}$ after transfection they were lysed with RIPA buffer (Sigma- 
Aldrich) containing Complete mini protease and phosphatase inhibitor cocktail tablets (Roche, Basel, Switzerland). Protein quantification was performed using the Bradford protein assay (Bio-Rad, Hercules, CA, USA) and $10 \mu \mathrm{g}$ of lysate was loaded per lane. Proteins were resolved by 8 or $10 \%$ sodium dodecyl sulfate-polyacrylamide gel electrophoresis gel and wet transferred to polyvinylidene difluoride membrane (EMD Millipore, Billerica, MA, USA). The signals were visualized by ECL Prime Western blotting Detection Reagent (Amersham, Piscataway, NJ, USA) and exposed to AGFA Curix X-ray film (AGFA, Mortsel, Belgium). The following $\mathrm{Ab}$ were used: antiHOXB9 (1:100, mouse, sc-398500) from Santa Cruz Biotechnology Inc. (Dallas, TX, USA) and anti-EREG (1:1000, rabbit, $12048 \mathrm{~S})$, anti-PARP (1:1000, rabbit, $9542 \mathrm{~S})$ and anti- $\beta$-tubulin (1:2000, rabbit, $2146 \mathrm{~S})$ from Cell Signaling Technology (Danvers, MA, USA).

\section{Generation of stable cell line overexpressing miR-215-5p}

Stable transfectants were generated using OriGene's pCMV6-Mir vectors with miR-215-5p precursor or control sequence and TurboFectin 8.0 (OriGene Technologies, Rockville, MD, USA). Stable clones were selected using $300 \mu \mathrm{g} \mathrm{ml}^{-1} \mathrm{G} 418$ (Sigma Aldrich). The stable expression of miR-215-5p was evaluated by RT-qPCR.

\section{In vivo tumorigenicity assay}

Five NSG mice (males, 8-10 weeks old, 21-26 g, initially obtained from The Jackson Laboratory, Bar Harbor, USA) were housed and monitored in individually ventilated cage system (Techniplast, Buguggiate, Italy) with ad libitum access to water and feeding. The assay was performed according to the protocol described previously ${ }^{46,47}$. Tumors have been palpable since day 14 and mice were sacrificed on day 25. During the experiment, tumor growth and animal behavior were individually monitored. Animal experiments were performed in accordance with national and EU animal welfare legislation and all procedures were approved by institutional (Masaryk University, Brno) and national ethics committees.

\section{Data normalization and statistical analyses}

The threshold cycle data were calculated by QuantStudio $12 \mathrm{~K}$ Flex software using the default threshold settings. All real-time PCR reactions were run in triplicates and average threshold cycle and SD values were calculated. The average expression levels of miR-215-5p in tumor and adjacent non-tumor tissues, as well as in the cell lines were normalized using RNU48 as a reference gene, the expression of EREG and HOXB9 was normalized using PMM1 (in case of tissue samples) or GAPDH (in case of cell lines) as a reference genes; subsequently, all data were transformed by the $2^{-\Delta \mathrm{Ct}}$ method. Statistical differences between the levels of miR-215-5p in tumor and non-tumor tissues were evaluated by the nonparametric Wilcoxon test for paired samples. Furthermore, Mann-Whitney $U$-test was used to analyze the correlation between miR-215-5p expression levels and clinical-pathological features of the patients. Survival analyses were performed using the log-rank test and Kaplan-Meier plots approach. For in vitro and in vivo analyses, the two-sided Student's $t$-test was used to compare the mean values between two groups. Data are presented as the mean values with SD unless otherwise noted (all in vitro measurements were repeated three times). All calculations were performed using GraphPad Prism version 5.00 (GraphPad Software, San Diego, CA, USA). $P$-values of $<0.05$ were considered statistically significant.

\section{Acknowledgements}

This work was supported by grant project GACR 16-18257S, the project CEITEC 2020 (LQ1601) provided by the Ministry of Education Youth and Sports of the Czech Republic and projects MUNI/A/1284/2015 and MUNI/11/InGA09/2014. The authors thank Helena Polakova, Andrej Besse and Jan Verner for their excellent technical assistance.

\section{Author details}

${ }^{1}$ Central European Institute of Technology, Masaryk University, Brno, Czech Republic. ${ }^{2}$ Department of Comprehensive Cancer Care, Masaryk Memorial Cancer Institute, Faculty of Medicine, Masaryk University, Brno, Czech Republic. ${ }^{3}$ Department of Pharmacology, Faculty of Medicine, Masaryk University, Brno, Czech Republic. ${ }^{4}$ Department of Clinical Analysis, Santa Lucia University Hospital, Cartagena, Spain. ${ }^{5}$ Department of Pathology, Santa Lucia University Hospital, Cartagena, Spain

\section{Competing interests}

The authors declare no competing financial interests.

Publisher's note: Springer Nature remains neutral with regard to jurisdictional claims in published maps and institutional affiliations.

\section{Supplementary information}

The online version of this article (doi:10.1038/s41389-017-0006-6) contains supplementary material.

Received: 8 May 2017 Revised: 16 September 2017 Accepted: 18 September 2017

Published online: 04 December 2017

\section{References}

1. Ferlay, J. et al. Cancer incidence and mortality worldwide: sources, methods and major patterns in GLOBOCAN 2012. Int. J. Cancer 136, E359-E386 (2015).

2. Sana, J., Faltejskova, P., Svoboda, M. \& Slaby, O. Novel classes of non-coding RNAs and cancer. J. Transl. Med. 10, 103 (2012).

3. Gomes, A. Q., Nolasco, S. \& Soares, H. Non-coding RNAs: multi-tasking molecules in the cell. Int. J. Mol. Sci. 14, 16010-16039 (2013).

4. Crea, F., Clermont, P. L., Parolia, A., Wang, Y. \& Helgason, C. D. The non-coding transcriptome as a dynamic regulator of cancer metastasis. Cancer Metastasis Rev. 33, 1-16 (2014).

5. Joh, R. I., Palmieri, C. M., Hill, I. T. \& Motamedi, M. Regulation of histone methylation by noncoding RNAs. Biochim. Biophys. Acta 1839, 1385-1394 (2014).

6. Lai, E. C. Micro RNAs are complementary to $3^{\prime}$ UTR sequence motifs that mediate negative post-transcriptional regulation. Nat. Genet. 30, 363-364 (2002). 
7. Cho, W. C. S. OncomiRs: the discovery and progress of microRNAs in cancers. Mol. Cancer 6, 60 (2007).

8. Calin, G. A. \& Croce, C. M. MicroRNA signatures in human cancers. Nat. Rev. Cancer 6, 857-866 (2006).

9. Slaby, O., Svoboda, M., Michalek, J. \& Vyzula, R. MicroRNAs in colorectal cancer: translation of molecular biology into clinical application. Mol. Cancer 8, 102 (2009).

10. Amankwatia, E. B. et al. MicroRNA-224 is associated with colorectal cancer progression and response to 5-fluorouracil-based chemotherapy by KRASdependent and -independent mechanisms. Br. J. Cancer 112, 1480-1490 (2015).

11. Faltejskova, P. et al. Identification and functional screening of microRNAs highly deregulated in colorectal cancer. J. Cell Mol. Med. 16, 2655-2666 (2012),

12. Necela, B. M., Carr, J. M., Asmann, Y. W. \& Thompson, E. A. Differential expression of microRNAs in tumors from chronically inflamed or genetic (APC (Min/+)) models of colon cancer. PLoS ONE 6, e18501 (2011).

13. Braun, C. J. et al. p53-Responsive micrornas 192 and 215 are capable of inducing cell cycle arrest. Cancer Res. 68, 10094-10104 (2008).

14. Song, B. et al. Molecular mechanism of chemoresistance by miR-215 in osteosarcoma and colon cancer cells. Mol. Cancer 9, 96 (2010).

15. Boni, V. et al. miR-192/miR-215 influence 5-fluorouracil resistance through cell cycle-mediated mechanisms complementary to its post-transcriptional thymidilate synthase regulation. Mol. Cancer Ther. 9, 2265-2275 (2010).

16. Li, S. et al. MicroRNA-215 inhibits relapse of colorectal cancer patients following radical surgery. Med. Oncol. Northwood Lond. Engl. 30, 549 (2013).

17. Agarwal, V., Bell G. W., Nam J.-W. \& Bartel, D.P. Predicting effective microRNA target sites in mammalian mRNAs. elife 4, e05005 (2015).

18. Maragkakis, M. et al. DIANA-microT Web server upgrade supports Fly and Worm miRNA target prediction and bibliographic miRNA to disease association. Nucleic Acids Res. 39, W145-W148 (2011)

19. Rehmsmeier, M., Steffen, P., Höchsmann, M. \& Giegerich, R. Fast and effective prediction of microRNA/target duplexes. RNA 10, 1507-1517 (2004).

20. Miranda, K. C. et al. A pattern-based method for the identification of MicroRNA binding sites and their corresponding heteroduplexes. Cell 126, 1203-1217 (2006).

21. John, B. et al. Human microRNA targets. PLoS Biol. 2, e363 (2004).

22. Riese, D. J. \& Cullum, R. L. Epiregulin: roles in normal physiology and cancer. Semin. Cell Dev. Biol. 28, 49-56 (2014).

23. Zhan, J. et al. Elevated HOXB9 expression promotes differentiation and predicts a favourable outcome in colon adenocarcinoma patients. Br. J. Cancer 11, 883-893 (2014).

24. Bonfrate, L. et al. MicroRNA in colorectal cancer: new perspectives for diagnosis, prognosis and treatment. J. Gastrointest. Liver Dis. 22, 311-320 (2013).

25. Hollis, M. et al. MicroRNAs potential utility in colon cancer: early detection, prognosis, and chemosensitivity. World J. Gastroenterol. 21, 8284-8292 (2015)

26. Stiegelbauer, $V$. et al. MicroRNAs as novel predictive biomarkers and therapeutic targets in colorectal cancer. World J. Gastroenterol. 20, 11727-11735 (2014).

27. Karaayvaz, M. et al. Prognostic significance of miR-215 in colon cancer. Clin. Colorectal. Cancer 10, 340-347 (2011).
28. Slattery, M. L. et al. An evaluation and replication of miRNAs with disease stage and colorectal cancer-specific mortality. Int. J. Cancer 137, 428-438 (2015).

29. Zhang, J. X. et al. Prognostic and predictive value of a microRNA signature in stage II colon cancer: a microRNA expression analysis. Lancet Oncol. 14, 1295-1306 (2013)

30. Yang, J. et al. Expression analysis of microRNA as prognostic biomarkers in colorectal cancer. Oncotarget 8, 52403-52412 (2017).

31. Georges, S. A. et al. Coordinated regulation of cell cycle transcripts by p53 inducible microRNAs, miR-192 and miR-215. Cancer Res. 68, 10105-10112 (2008).

32. Banks, D. et al. L2DTL/CDT2 and PCNA interact with p53 and regulate p53 polyubiquitination and protein stability through MDM2 and CUL4AVDB1 complexes. Cell Cycle (Georgetown, TX) 5, 1719-1729 (2006)

33. Jeanes, A., Gottardi, C. J. \& Yap, A. S. Cadherins and cancer: how does cadherin dysfunction promote tumor progression? Oncogene 27, 6920-6929 (2008).

34. Nechiporuk, T., Fernandez, T. E. \& Vasioukhin, V. Failure of epithelial tube maintenance causes hydrocephalus and renal cysts in Dlg5 $5^{-1-}$ mice. Dev. Cell 13, 338-350 (2007).

35. Ge, G. et al. miR-215 functions as a tumor suppressor in epithelial ovarian cancer through regulation of the X-chromosome-linked inhibitor of apoptosis. Oncol. Rep. 35, 1816-1822 (2016).

36. Ye, M. et al. Curcumin promotes apoptosis by activating the p53-miR-192-5p/ 215-XIAP pathway in non-small cell lung cancer. Cancer Lett. 357, 196-205 (2015).

37. White, N. M. A. et al. mRNA profiling in metastatic renal cell carcinoma reveals a tumour-suppressor effect for miR-215. Br. J. Cancer 105, 1741-1749 (2011).

38. Hou, $Y$. et al. miR-215 functions as a tumor suppressor and directly targets ZEB2 in human non-small cell lung cancer. Oncol. Lett. 10, 1985-1992 (2015).

39. Li, Q. W. et al. MicroRNA-215 functions as a tumor suppressor and directly targets ZEB2 in human pancreatic cancer. Genet. Mol. Res. 14, 16133-16145 (2015).

40. Huang, K. et al. Overexpression of HOXB9 promotes metastasis and indicates poor prognosis in colon cancer. Chin. J. Cancer Res. 26, 72-80 (2014).

41. Shrestha, B. et al. Homeodomain containing protein HOXB9 regulates expression of growth and angiogenic factors, facilitates tumor growth in vitro and is overexpressed in breast cancer tissue. FEBS J. 279, 3715-3726 (2012).

42. Hayashida, T. et al. HOXB9, a gene overexpressed in breast cancer, promotes tumorigenicity and lung metastasis. Proc. Natl. Acad. Sci. USA 107, 1100-1105 (2010).

43. Wu, S. Y. et al. A miR-192-EGR1-HOXB9 regulatory network controls the angiogenic switch in cancer. Nat. Commun. 7, 11169 (2016).

44. Song, B. et al. miR-192 Regulates dihydrofolate reductase and cellular proliferation through the p53 microRNA circuit. Clin. Cancer Res. 14, 8080-8086 (2008).

45. Bunz, F. et al. Requirement for p53 and p21 to sustain G2 arrest after DNA damage. Science 282, 1497-1501 (1998)

46. Morton, C. L. \& Houghton, P. J. Establishment of human tumor xenografts in immunodeficient mice. Nat. Protoc. 2, 247-250 (2007).

47. McKay, J. A. et al. Evaluation of the epidermal growth factor receptor (EGFR) in colorectal tumours and lymph node metastases. Eur. J. Cancer 38, 2258-2264 (2002). 